\title{
Cerebral Ischemic Complications of Original Subclavian Artery Stenting: Relationship between Lesion Location and Procedural Strategy
}

Masayasu Arihara, ${ }^{1}$ Kei Miyata, ${ }^{1,2}$ Satoshi lihoshi, ${ }^{1}$ Rintaro Yokoyama, ${ }^{1}$ Ayumu Yamaoka, ${ }^{1}$ Yuto Suzuki, ${ }^{1}$ Takeshi Mikami, ${ }^{1}$ Masahiko Wanibuchi, ${ }^{1}$ and Nobuhiro Mikuni ${ }^{1}$

Objective: Clinical research demonstrating safety and security in subclavian artery stenting (SAS) is limited because treatment strategies for SAS vary greatly among institutions, operators, and clinical conditions, as no standard protocol exists. As stenotic lesions frequently occur in the proximal portion of the subclavian artery (SA), it is often difficult to stabilize the guiding catheter for stent delivery. Our purpose was to assess whether lesion location can be used to predict optimal procedural strategies.

Methods: We performed a retrospective, single-center observational study on 26 consecutive patients who underwent SAS between 2004 and 2016. We categorized patients treated with SAS into two groups according to lesion location: those with lesions located less than $10 \mathrm{~mm}$ from the origin of the SA (Group P) and those with lesions in the distal portion (Group D). We defined a bidirectional approach via both brachial and femoral puncture as well as bilateral brachial puncture as combined access. In pull-through method, a guidewire was passed from one sheath to another. We compared Group P with Group D in terms of procedural strategy and efficacy.

Results: All 26 cases were clinically successful. Asymptomatic cerebral embolism was found in 10 (48\%) of 21 cases in which postoperative MRI was performed, and the number of spotty lesions was less than four in every patient. Arterial access-site complications were documented in eight patients. Two cases of late in-stent restenosis were retreated with balloon dilatation. Cases with retrograde ipsilateral vertebral artery (VA) blood flow and symptomatic lesions occurred more frequently in Group P than in Group D ( $79 \%$ vs. $42 \% ; p=0.06,71 \%$ vs. $33 \% ; p=0.05$, respectively). Patients in Group P were significantly more often treated with combined access ( $100 \%$ vs. $42 \% ; p=0.001)$, with the pull-through method $(64 \%$ vs. $0 \% ; p<0.001)$ than those in Group $D$. The distal end of the stent was involved with the ipsilateral VA orifice more often in Group D than in Group P (50\% vs. $7 \%$; $p=0.02)$.

Conclusion: These results demonstrate that our strategy is a safe and effective method of stenting for SA stenosis in the portion of the artery that is proximal to the aorta. We should pay special attention to the risk of arterial access-site complications.

Keywords > subclavian artery occlusive disease, stenting, endovascular therapy, cerebral embolism

\footnotetext{
${ }^{1}$ Department of Neurosurgery, Sapporo Medical University School of Medicine, Sapporo, Hokkaido, Japan

${ }^{2}$ Department of Emergency Medicine, Sapporo Medical University School of Medicine, Sapporo, Hokkaido, Japan
}

Received: May 13, 2019; Accepted: July 15, 2019

Corresponding author: Kei Miyata. Department of Neurosurgery, Sapporo Medical University School of Medicine, South-1st, West-16th, Chuo-ku, Sapporo, Hokkaido 060-8543, Japan Email:miyata@sapmed.ac.jp

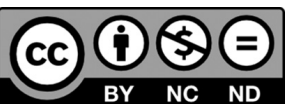

This work is licensed under a Creative Commons Attribution-NonCommercialNoDerivatives International License.

(C)2019 The Japanese Society for Neuroendovascular Therapy

\section{Introduction}

Subclavian artery occlusive disease (SAOD), which has a reported prevalence of $0.5-2 \%$ in the general population, ${ }^{1-3)}$ can lead to vertebrobasilar ischemia, upper extremity ischemia, and coronary subclavian steal syndrome (CSSS) in patients who have received an internal mammary artery graft bypass. ${ }^{4,5)}$ Recent studies have reported that endovascular treatment (EVT), an alternative to surgical revascularization for SAOD, yields long-term good outcomes with comparable success rates. ${ }^{6-9)}$ Subclavian artery stenting (SAS) has been shown to have lower periprocedural complication rates of 3-6\%, ${ }^{10-13)}$ and specifically a lower cerebral 
ischemic complication rate of only 0-4\%. ${ }^{6,14-16)}$ In addition, treatment strategies for SAS vary greatly among institutions, operators, and clinical conditions, as no standard protocol exists. ${ }^{17)}$ As stenotic lesions often occur in the subclavian artery (SA) proximal to the aortic arch, ${ }^{16)}$ there is frequently some difficulty in stabilizing the guiding catheter (GC) position and deploying the stent. It seems likely that lesion location could be useful in determining optimal procedural strategies and identifying the most probable complications, yet the available literature on this topic is very scant. To explore this possibility, we evaluated the safety and efficacy of SAS for SAOD with particular attention to the effect of lesion location on the outcomes of various procedures.

\section{Materials and Methods}

\section{Study design and patients}

This retrospective, observational study was conducted at Department of Neurosurgery, Sapporo Medical University. Eligible subjects were 30 SAOD patients treated with EVT between January 1, 2004 and December 31, 2016. Asymptomatic patients were eligible if their upper extremities had a difference in systolic blood pressure of at least $40 \mathrm{mmHg}$. Eligibility criteria for symptomatic patients included hemodynamically unstable stenosis presenting as vertebrobasilar blood flow deficiency, upper limb ischemia due to subclavian steal syndrome (SSS), or coronary artery ischemia after internal mammary artery graft bypass, referred to as CSSS.

From among the 30 cases that met these criteria, we excluded two cases with lesions in the brachiocephalic artery and another two cases with in-stent restenosis.

\section{Ethics approval}

This study was approved by the research ethics committee of Sapporo Medical University. We obtained patient consent and referral documents from each patient's primary hospital or institution with the stipulation that we would disclose only the data that was necessary for our study and that we would not disclose any personal information or other items that the hospital did not wish to be disclosed regarding medical care information (e.g., official medical charts).

\section{Definition of variables}

Clinical data were collected from electronic medical records, operative notes, and radiology records. We classified patients into two groups according to the distance from origin of the SA to the proximal side of the lesion using 3D-CT angiogram: lesions beginning less than $10 \mathrm{~mm}$ from

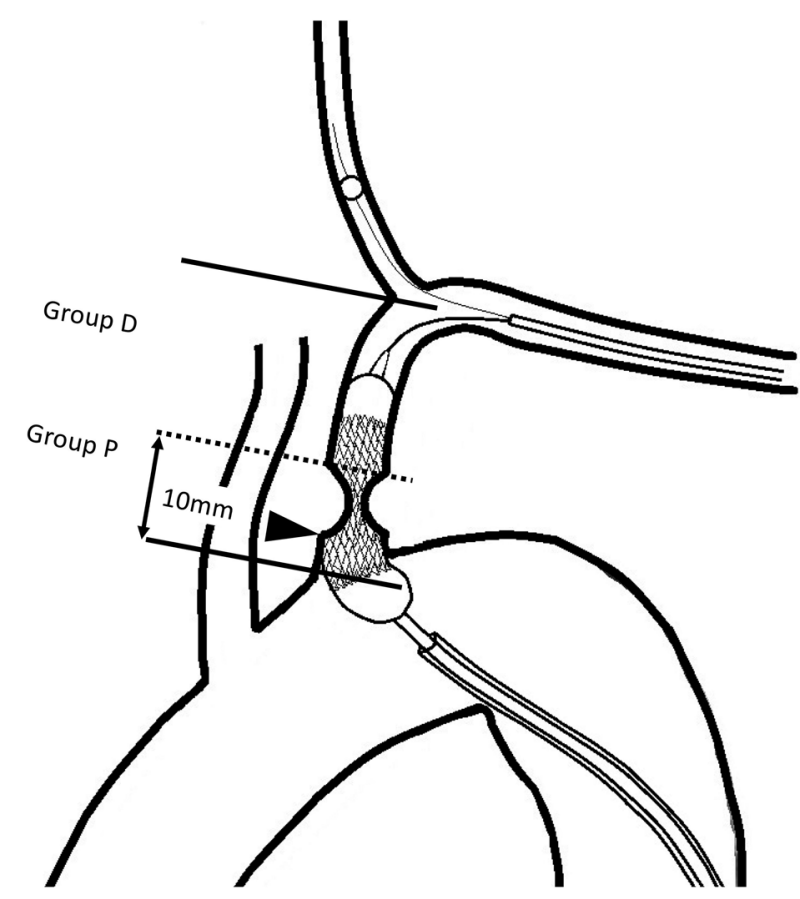

Fig. 1 Depiction of our combined access strategy with the pullthrough method permitting bidirectional access via femoral and brachial punctures with extraction of a guidewire from one sheath to another. Classification of the lesion: Group P includes lesions with the proximal edge located less than $10 \mathrm{~mm}$ from the origin of the SA and Group D includes lesions beginning at a more distal location. The picture is an example of a Group P case with the proximal edge of the narrowing (arrowhead) in the proximal SA origin to the orifice of the VA. SA: subclavian artery; VA: vertebral artery

the origin of the SA, that is, lesions in the proximal $10 \mathrm{~mm}$ (Group P), and those beginning more than $10 \mathrm{~mm}$ from the origin of the SA, that is, lesions in the distal portion (Group D) (Fig. 1). Neurological classification (symptomatic or asymptomatic), side of the lesion (left or right), calcification (occupying more or less than one-third of the diameter as assessed by multi-detector CT), degree of stenosis (\%) (pre- and post-stenting), length of the lesion $(\mathrm{mm})$, distance from origin of the SA to the proximal side of the lesion, and distance from the distal side of the lesion to the orifice of the ipsilateral vertebral artery (VA) (mm) were assessed.

Radiological examination included head computed tomography (CT) and MRI within the first few days after the procedure. We ourselves assessed new positive spots on diffusion-weighted MRI (DW-spots), the number and distributions of these spots, and other adverse events.

\section{SAS procedures}

Either clopidogrel $75 \mathrm{mg}$ and aspirin $100 \mathrm{mg}$ or cilostazol $200 \mathrm{mg}$ was administered daily starting 5 days before the 


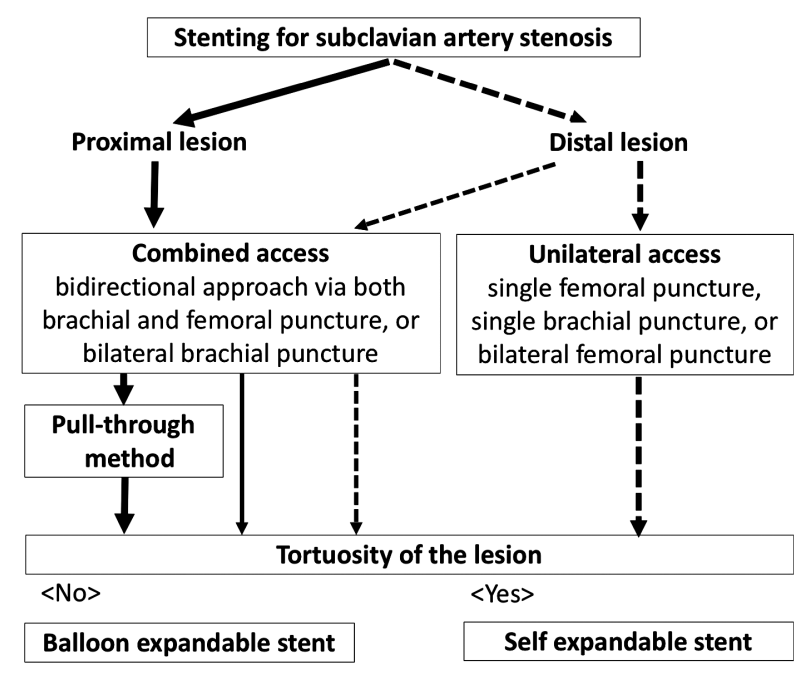

Fig. 2 Flowchart of strategy selection for SAS. Combined access indicates both femoral and brachial artery punctures or bilateral brachial artery punctures, and pull-through method indicates extraction of a guidewire from one sheath to another. Unilateral access indicates single femoral puncture, single brachial puncture, or bilateral femoral puncture. SAS: subclavian artery stenting

procedure. Three neuro-interventional doctors qualified by the Japanese Society of Neuroendovascular Therapy performed the procedures under local anesthesia. The patients were administered 3000-5000 units of unfractionated heparin to maintain activated clotting time $250-300$ seconds.

The flow diagram of our SAS procedures is shown in Fig. 2. We classified patients as "combined access" or "unilateral access" according to their arterial puncture sites. We defined a bidirectional approach via both brachial and femoral puncture as well as bilateral brachial puncture as "combined access." In contrast, "unilateral access" was defined as single femoral puncture, single brachial puncture, or bilateral femoral puncture. The latter approach was selected in cases with an absent pulse in the brachial artery or severe arteriosclerosis obliterans in the lower extremities. An 8 Fr GC or a 6 Fr guiding sheath was deployed into the descending aortic aorta or the SA for the stent delivery.

In cases with the lesion located in the proximal portion of the SA or cases of chronic total occlusion, we introduced a "pull-through method" to augment the stabilizing wire tension and stabilization of the GC (Figs. 2 and 3). ${ }^{18)} \mathrm{We}$ advanced a $300 \mathrm{~cm}$ 0.035-inch guidewire from one introducer sheath to the lesion and then across the lesion. The guidewire was passed from the brachial guiding sheath to the femoral sheath via direct insertion and was caught outside of the body. Subsequent procedures including balloon dilatation and/or stent deployment were performed using the guidewire.
Except in cases with hypoplastic VA, access difficulty or apparent retrograde VA flow due to SSS, a distal embolic protection device (EPD), specifically, either a Percusurge Guardwire system (Covidien, Minneapolis, MN, USA) (Fig. 3) or a 6-French temporary occlusion catheter (Fig. 4) was routinely prepared.

A balloon expandable stent (BE) was placed in the proximal portion of the SA to enable easy positioning and sufficient expansion force. In contrast, a self-expandable stent (SE) was selected for lesions with vessel tortuosity and lesions that were located near the orifice of the VA. Pre-balloon dilatation was performed in nearly all cases; in cases of mild stenosis, however, direct stenting was performed. For residual stenosis after stenting, post-balloon dilatation was added with a goal of $30 \%$ stenosis.

The hemostasis methods in the femoral puncture area included vascular closure devices or manual compression. The method used in the brachial puncture area was either manual compression or a new method that we introduced in 2012 that consists of staged compression after substituting a large guiding sheath into a smaller introducer kit. Administration of heparin was reversed by 10-20 mg of protamine sulfate before the hemostasis. Anticoagulation included continuous intravenous infusion of heparin or argatroban by infusion pump within 24 hours after SAS. Initial clinical success was defined as relief or substantial reduction of the preprocedural symptoms, as listed in Table 1. In-stent restenosis was defined as more than $60 \%$ lumen reduction with or without renewed clinical symptoms.

\section{Statistical analysis}

We compared anatomical characteristics, procedural methods, and DW-spots between Group P and Group D. In addition, we examined the relation between the use of EPD, the selected approach route, and DW-spots. Continuous variables were expressed as mean, standard deviation (SD), or, where indicated, median and interquartile range (IQR). Categorical data were expressed as numbers and percentages. We used $\chi^{2}$ tests for categorical data, except when expected cells were found to be less than 5, in which case we used Fisher's exact test (one-sided test). The statistical significance of differences in mean values between two populations was assessed with Student's t-test if variances were equal as determined by an F test, or otherwise with Welch's t-test. The median value comparison was evaluated using the Mann-Whitney U test for parametric analysis. All statistical analysis was performed using SPSS version 22 (SPSS Inc., Chicago, IL, USA). A p value $<0.05$ was considered to 

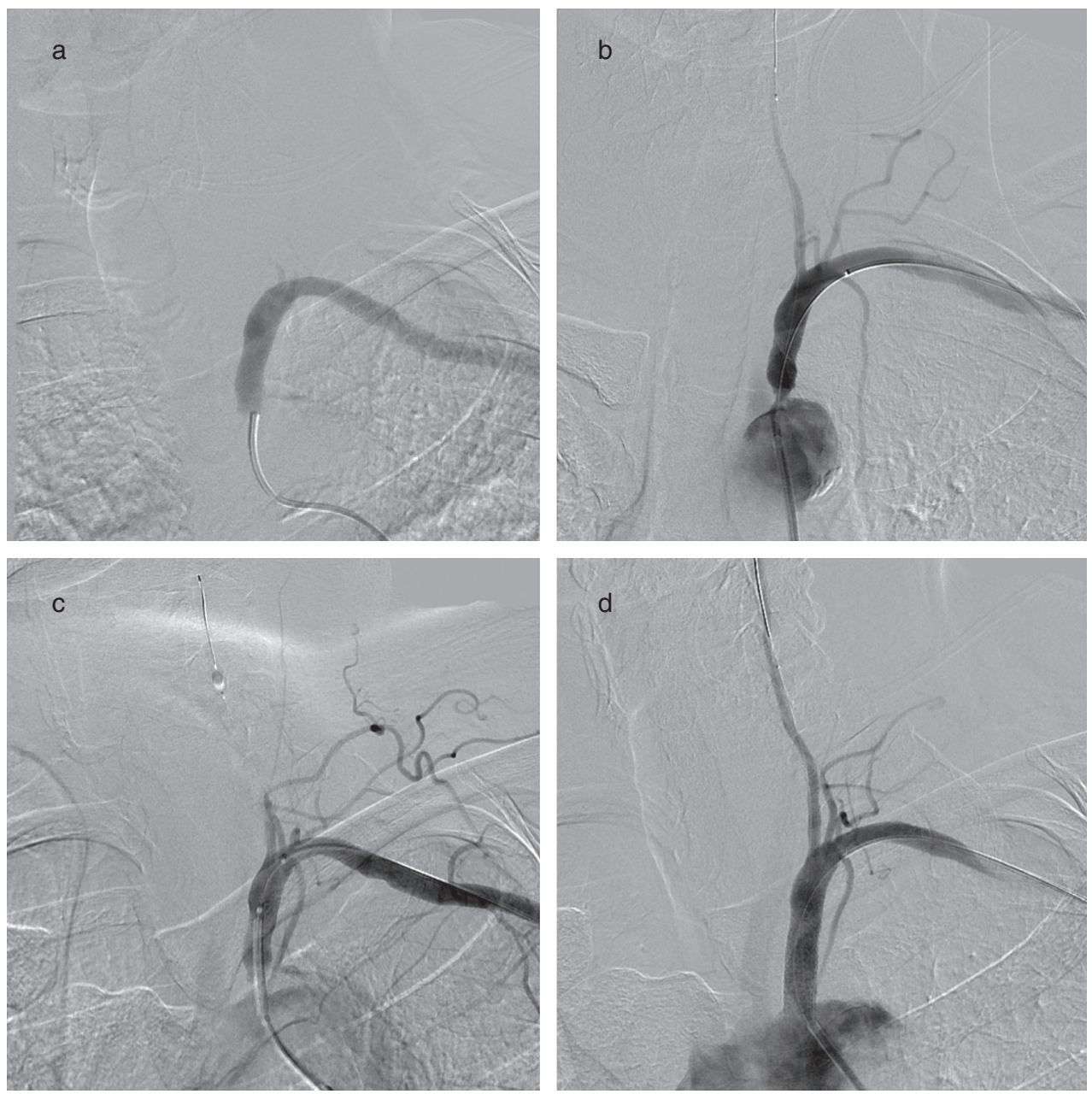

Fig. 3 Example of the pull-through method which improves GC stabilization and successful stent placement. (a) Preoperative left SA angiography in a LAO view showing disappearance of the anterograde VA blood flow. (b) Brachial SA angiography in an anterior view showing a stenosis at the proximal portion of the SA and the VA, with a temporary balloon occlusion catheter advanced into the left VA. (c) Brachial SA angiography showing adequate positioning of a balloon expanding stent under left VA protection and the pull-through method. (d) Postoperative angiography in a LAO view showing adequate dilatation and left VA recanalization. GC: guiding catheter; LAO: left anterior oblique; SA: subclavian artery; VA: vertebral artery

indicate significance while $\mathrm{p}<0.2$ was considered to indicate a non-significant trend.

\section{Results}

The backgrounds of the patients are provided in Table 1. All 26 cases were considered clinically successful. No deaths or morbidity occurred within the 30-day perioperative period. We experienced two patients with asymptomatic and symptomatic in-stent restenosis and promptly retreated both of them with balloon dilatation (10 months and 15 months after the procedure, respectively).

As Table 2 shows, cases with retrograde ipsilateral VA blood flow and symptomatic lesions were more frequently observed in Group P than in Group D (79\% vs. $42 \%$; $p=$ $0.06,71 \%$ vs. $33 \% ; p=0.05$, respectively). Preoperative severity of stenosis was statistically higher in Group P than in Group D (90\% vs. $80 \% ; p=0.005)$. Patients in Group P were significantly more often treated with combined access $(100 \%$ vs. $42 \% ; \mathrm{p}=0.001)$ with pull-through method $(64 \%$ vs. $0 \% ; \mathrm{p}<0.001)$ and $\mathrm{BE}(71 \%$ vs. $17 \%$; $\mathrm{p}=0.005)$ than those in Group D. The distal end of the stent was involved on the ipsilateral VA orifice significantly more often in Group D than in Group P (7\% vs. $50 \% ; \mathrm{p}=0.02)$.

Access-site complications were documented in eight patients. These included two major complications, namely, femoral artery pseudoaneurysm requiring operative 

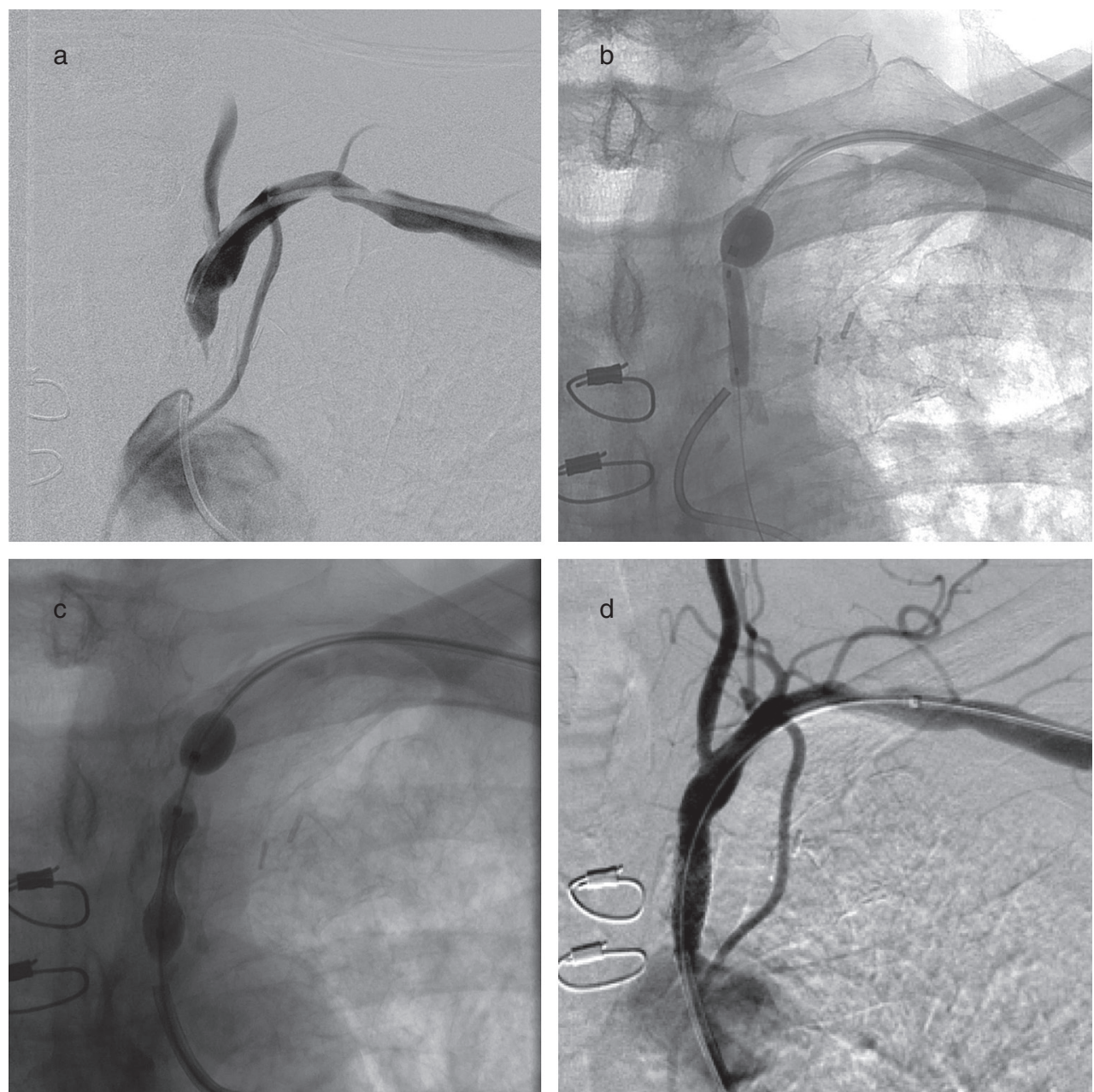

Fig. 4 Example of the pull-through method with a temporary occlusion BGC for a CSSS patient. (a) Preoperative brachial SA angiography in an anterior view showing a chronic total occlusion and subclavian steal with slow flow in the left internal mammary artery. (b) Fluoroscopic image in an anterior view showing pre-balloon dilatation through the guidewire introduced from the left brachial artery. (c) Fluoroscopic image in an anterior view showing placement of a BE with the pull-through method. (d) Postoperative aortogram in an anterior view showing sufficient recanalization of the left SA. BGC: balloon guiding catheter; BE: balloon expandable stent; CSSS: coronary subclavian steal syndrome; SA: subclavian artery

management and brachial artery pseudo-occlusion treated with non-operative management.

In 21 cases, postoperative MRI was performed ( 11 cases in Group P and 10 cases in Group D). DW-spots were found in 10 patients $(48 \%)$, and the number of DW-spots was less than four in every patient. None of the new lesions were symptomatic with regard to neurological deficits. In four cases, DW-spots were documented in both the anterior and posterior circulation; one of these was treated with single brachial access while the others were treated with combined access. The incidence of new DW-lesions was not significantly different between Group P and Group D (36\% vs. $60 \%, \mathrm{p}=0.3)$.

An EPD was introduced in 17 cases $(65 \%)$ for stroke prevention, including 15 cases of distal EPD and 2 cases of balloon guiding catheter (BGC). The rate of treatment with EPD tended to be higher in combined access than in unilateral access ( $78 \%$ vs. $38 \%, \mathrm{p}=0.07)$. However, no specific tendency was seen regarding the relation between EPD use and the occurrence of DW-spots (EPD use group, 54\% vs. non-EPD use group, $38 \%, \mathrm{p}=0.4$ ).

\section{Discussion}

We investigated the associations between lesion location and procedural variation. Our results demonstrate that our strategy is a safe and effective method of SAS in the proximal portion of the SA. It also shows that we should pay special attention to the risk of arterial access-site complications. 
Table 1 Patient backgrounds

\begin{tabular}{ll} 
Patient characteristics & $\mathrm{n}(\%)$ \\
Male sex & $20(77)$ \\
Age (years) & \\
Mean, SD & $67,7.4$ \\
Median (IQR) & $67(62-74)$ \\
Left side & $23(88)$ \\
Symptomatic & $14(54)$ \\
CSSS & 3 \\
Upper arm ischemia & 5 \\
Syncope & 1 \\
Dizziness & 3 \\
Stroke & 2 \\
Subclavian steal syndrome & $16(62)$ \\
Chronic total occlusion & $3(12)$ \\
Severity of stenosis & \\
Mean, SD & 84,11 \\
Median (IQR) & $85(80-90)$ \\
Calcification of the lesion & $13(50)$ \\
Coronary artery disease & $10(38)$ \\
Post CABG & 5 \\
Indication of PCl & 1 \\
Post PCI & 3 \\
Angina & 1 \\
\hline
\end{tabular}

CABG: coronary artery bypass graft; CSSS: coronary subclavian steal syndrome; IQR: interquartile range; PCl: percutaneous coronary intervention; SD: standard deviation

In cases where SAOD occurred in the proximal portion of the SA, we introduced combined access with the pull-through method. In the pull-through method, which allows for greater stabilization of the device, a guidewire is passed through from one sheath to another across the stenosis or obstructive lesion. ${ }^{19)}$ In the cerebrovascular region, this technique is applied to lesions in the subclavian and brachiocephalic arteries and at the origin of the VA. ${ }^{20,21)}$ Stabilization of the GC may also help ensure appropriate placement of the BE into the proximal portion of the SA. The pull-through method may also aid in positioning of the GCs while allowing for sufficient pushability and stability when the BE needs to be covered by the GC at the lesion location to avoid dropout of the stent, or when the deflated balloon is difficult to extract from the stent-mesh.

On the other hand, combined access has the following disadvantages. Arterial puncture on the brachial side may be difficult, especially when the pulsatility is weak. The pull-through method also makes the overall procedure more complicated as it requires additional procedures such as holding and withdrawing the guidewire using the gooseneck snare wire. The risk of thromboembolism may increase as multiple devices are placed into the blood vessel.

The high incidence of local complications remains a significant limitation of the transluminal brachial approach.
As reported previously, local complications such as infection, pseudoaneurysm, thrombosis, and hematoma are seen in approximately $5 \%{ }^{22,23)}$ of procedures, and a greater number of complications were observed with the brachial approach than with the femoral approach. $22,24,25)$ The use of long sheaths has been described as a risk factor for upper-arm access complications, which occur in $1-6.5 \%$ of coronary or peripheral artery interventions. ${ }^{26,27)}$ Because we inserted large long sheaths into the brachial artery, our series showed a higher incidence of local complications than previous reports have shown. In the latter half of our series, however, our new method of staged manual compression completely abolished any local complications involving brachial hematoma.

We consider that our procedure is safe and acceptable because there were few DW-spots and no symptomatic lesions in this series. Our results show higher rates of new cerebral ischemic lesions after the SAS procedure as compared with previous reports, ${ }^{6,14-16,28)}$ but this is probably because we obtained more detailed images than previous studies could acquire and because we searched for these spots very thoroughly. The incidence of such ischemic events may also have been lower in previous reports because ipsilateral VA flow was much lower than SA flow, or, in cases of proximal SAOD, even reversed. ${ }^{29)}$ The incidence of DW-spots was not significantly different between Groups $\mathrm{P}$ and D, although asymptomatic ischemic lesions were detected in half of the patients in Group D. When the lesion is close to the orifice of the ipsilateral VA, stentmesh coverage of the VA orifice or protrusion of plaque may cause distal embolism via the VA.

EPD was more frequently used in combined access than in unilateral access. In previous reports, it has been unclear whether EPD use might contribute to the reduction of ischemic complications. ${ }^{14,30)}$ However, antegrade VA blood flow may recover immediately following percutaneous transluminal angioplasty for SSS. We speculate that EPD might compensate for the potential additional risks associated with using complicated techniques such as combined access and the pull-through method, which have higher adverse event rates compared with unilateral access.

Several limitations of this study should be acknowledged. First, the non-random nature of the study and the limitations of our statistical methods must be mentioned. We deliberately modified each item, from quantitative data to categorical variables, and these classifications may have affected the results. As other variables that may significantly affect 
Table 2 Comparison of anatomical characteristics, procedural methods, and outcomes in each group

\begin{tabular}{|c|c|c|c|c|}
\hline Patient characteristics & & $\begin{array}{c}\text { Group P } \\
\mathrm{N}=14(\%)\end{array}$ & $\begin{array}{c}\text { Group D } \\
\mathrm{N}=12(\%)\end{array}$ & $p$ value \\
\hline Male sex & & $9(64)$ & $11(92)$ & 0.1 \\
\hline \multicolumn{5}{|l|}{ Age (years) } \\
\hline & Mean, SD & $68,6.4$ & $66,8.7$ & 0.5 \\
\hline & Median (IQR) & $68(63-74)$ & $65(59-74)$ & \\
\hline Symptomatic & & $10(71)$ & $4(33)$ & 0.05 \\
\hline Angiographic retrograde VA flow & & $11(79)$ & $5(42)$ & 0.06 \\
\hline \multicolumn{5}{|l|}{ Severity of the lesion (\%) } \\
\hline Pre & Median (IQR) & $90(80-96)$ & $80(64-88)$ & 0.005 \\
\hline Post & Median (IQR) & $10(4-16)$ & $10(5-20)$ & 0.4 \\
\hline \multicolumn{5}{|l|}{ Lesion (mm) } \\
\hline Length of lesion & Mean, SD & $12(8.3-21)$ & $8.5(6.5-16)$ & 0.5 \\
\hline Distance from the aorta to the lesion & Median (IQR) & $7(2-9.5) 6,4$ & $33(23-38)$ & $<0.001$ \\
\hline Distance from the lesion to VA origin & Median (IQR) & $23(14-35)$ & $8.8(5.9-13)$ & 0.01 \\
\hline Ipsilateral VA diameter & Median (IQR) & $4(3.1-4.5)$ & $4.5(2.7-5.6)$ & 0.5 \\
\hline \multicolumn{5}{|l|}{ Procedure } \\
\hline Combined access & & $14(100)$ & $5(42)$ & 0.001 \\
\hline with pull-through method & & $9(64)$ & $0(0)$ & $<0.001$ \\
\hline Unilateral access & & $0(0)$ & 7 (58) Fe:4, Br:3 & 0.001 \\
\hline Balloon expandable stent & & $10(71)$ & $2(17)$ & 0.005 \\
\hline Embolic protection for ipsilateral VA & & $10(71)$ & $7(58)$ & 0.4 \\
\hline Stent-mesh coverage of the ipsilateral VA orifice & & $1(7)$ & $6(50)$ & 0.02 \\
\hline
\end{tabular}

Br: brachial; Fe: femoral; IQR: interquartile range; SD: standard deviation; VA: vertebral artery

outcomes are not recorded in this study, confounding factors cannot be eliminated, and selection bias is perhaps inevitable. For example, several factors related to arteriosclerosis, such as hypertension, diabetes mellitus, dyslipidemia, and smoking, as well as procedure duration was not individually recorded. Second, advances in endovascular device design, changes in procedure strategy, and learning curve of operators over the decade during which we performed the procedures reported here should be considered. Third, this study is a single-center retrospective study. Given the small number of cases, we determined that univariate analysis was optimal for our study. In order to further clarify risk factors for embolic events and the selection of patients for EPD, we will need to accumulate larger numbers of cases and evaluate them using multivariate analysis.

\section{Conclusion}

We investigated the relation of lesion location with procedural strategy and efficacy in SAS. Our study demonstrated that our strategy for pre-vertebral SA is safe and effective, offering a high clinical success rate and a low major complication rate. However, we should pay special attention to the risk of access-site complications.

\section{Disclosure Statement}

There is no conflict of interest to be disclosed regarding this article.

\section{References}

1) Perrault LP, Carrier M, Hudon G, et al: Transluminal angioplasty of the subclavian artery in patients with internal mammary grafts. Ann Thorac Surg 1993; 56: 927-930.

2) Sixt S, Rastan A, Schwarzwälder U, et al: Results after balloon angioplasty or stenting of atherosclerotic subclavian artery obstruction. Catheter Cardiovasc Interv 2009; 73: 395-403.

3) Shadman R, Criqui MH, Bundens WP, et al: Subclavian artery stenosis: prevalence, risk factors, and association with cardiovascular diseases. J Am Coll Cardiol 2004; 44: 618-623.

4) Takach TJ, Reul GJ, Cooley DA, et al: Myocardial thievery: the coronary-subclavian steal syndrome. Ann Thorac Surg 2006; 81: 386-392.

5) Tortoledo F, Sánchez A, Izaguirre L, et al: Endovascular repair of symptomatic coronary-subclavian steal syndrome due to stenosis of the proximal left subclavian artery. Cardiol Rev 2005; 13: 128-129. 
6) Henry M, Amor M, Henry I, et al: Percutaneous transluminal angioplasty of the subclavian arteries. J Endovasc Surg 1999; 6: 33-41.

7) Rodriguez-Lopez JA, Werner A, Martinez R, et al: Stenting for atherosclerotic occlusive disease of the subclavian artery. Ann Vasc Surg 1999; 13: 254-260.

8) Cinà CS, Safar HA, Laganà A, et al: Subclavian carotid transposition and bypass grafting: consecutive cohort study and systematic review. J Vasc Surg 2002; 35: 422-429.

9) Salman R, Hornsby J, Wright LJ, et al: Treatment of subclavian artery stenosis: a case series. Int J Surg Case Rep 2016; 19: 69-74.

10) Millaire A, Trinca M, Marache $P$, et al: Subclavian angioplasty: immediate and late results in 50 patients. Cathet Cardiovasc Diagn 1993; 29: 8-17.

11) Dorros G, Lewin RF, Jamnadas $P$, et al: Peripheral transluminal angioplasty of the subclavian and innominate arteries utilizing the brachial approach: acute outcome and follow-up. Cathet Cardiovasc Diagn 1990; 19: 71-76.

12) Hebrang A, Maskovic J, Tomac B: Percutaneous transluminal angioplasty of the subclavian arteries: long-term results in 52 patients. AJR Am J Roentgenol 1991; 156: 1091-1094.

13) Patel SN, White CJ, Collins TJ, et al: Catheter-based treatment of the subclavian and innominate arteries. Catheter Cardiovasc Interv 2008; 71: 963-968.

14) Michael TT, Banerjee S, Brilakis E: Subclavian artery intervention with vertebral embolic protection. Catheter Cardiovasc Interv 2009; 74: 22-25.

15) Jain SP, Ramee SR, Ansel GM, et al: Endoluminal stenting of subclavian and innominate artery: acute and long term results from a multicenter stent registry. Circulation 1998; 98: 484.

16) Palchik E, Bakken AM, Wolford HY, et al: Subclavian artery revascularization: an outcome analysis based on mode of therapy and presenting symptoms. Ann Vasc Surg 2008; 22: 70-78.

17) Turgeman Y, Suleiman K, Feldman A, et al: Transradial diagnosis and intervention of supraaortic vessels. J Invasive Cardiol 2013; 25: 300-303.

18) Matsumoto Y, Nakai K, Tsutsumi M, et al: A simplified pull-through angioplasty technique without a snare device. A technical note. Interv Neuroradiol 2013; 19: 167-172.

19) Ginsburg R, Thorpe P, Bowles CR, et al: Pull-through approach to percutaneous angioplasty of totally occluded common iliac arteries. Radiology 1989; 172: 111-113.

20) Harada K, Nakahara I, Tanaka M, et al: [Therapeutic strategy and outcome of stenting for subclavian and innominate artery occlusive disease]. No Shinkei Geka 2004; 32: 151-158. (in Japanese)

21) Yamamoto $T$, Ohshima $T$, Ishikawa $K$, et al: Feasibility and safety of distal and proximal combined endovascular approach with a balloon-guiding catheter for subclavian artery total occlusion. World Neurosurg 2017; 100: 709.e5-709.e9.

22) Berger L, Bouziane Z, Felisaz A, et al: Long-term results of 81 prevertebral subclavian artery angioplasties: a 26-year experience. Ann Vasc Surg 2011; 25: 1043-1049.

23) de Vries JP, Jager LC, van den Berg JC, et al: Durability of percutaneous transluminal angioplasty for obstructive lesions of proximal subclavian artery: long-term results. J Vasc Surg 2005; 41: 19-23.

24) Amor M, Eid-Lidt G, Chati Z, et al: Endovascular treatment of the subclavian artery: stent implantation with or without predilatation. Catheter Cardiovasc Interv 2004; 63: 364-370.

25) Alkhouli M, Porter J, Waits B, et al: Distal embolization during percutaneous subclavian artery intervention. Vasc Endovascular Surg 2016; 50: 175-179.

26) Alvarez-Tostado JA, Moise MA, Bena JF, et al: The brachial artery: a critical access for endovascular procedures. J Vasc Surg 2009; 49: 378-385; discussion 385.

27) Stavroulakis K, Usai MV, Torsello G, et al: Efficacy and safety of transbrachial access for iliac endovascular interventions. J Endovasc Ther 2016; 23: 454-460.

28) Soga $Y$, Tomoi $Y$, Fujihara M, et al: Perioperative and longterm outcomes of endovascular treatment for subclavian artery disease from a large multicenter registry. $J$ Endovasc Ther 2015; 22: 626-633.

29) Ringelstein EB, Zeumer H: Delayed reversal of vertebral artery blood flow following percutaneous transluminal angioplasty for subclavian steal syndrome. Neuroradiology 1984; 26: 189-198.

30) Yokoi Y: Vertebral embolic protection: the final answer for protection of subclavian artery intervention? Catheter Cardiovasc Interv 2009; 74: 26. 